\title{
Feeding behavior in sexual and clonal strains of Poeciliopsis
}

\author{
Stephen C. Weeks *, Oscar E. Gaggiotti, Russell A. Schenck **, Kurt P. Spindler ***, and Robert C. Vrijenhoek \\ Center for Theoretical and Applied Genetics, Rutgers University, PO Box 231, New Brunswick, NJ 08903, USA
}

Recepted January 22, 1991 / Accepted October 1, 1991

\begin{abstract}
Summary. Sexual and clonal fish of the genus Poeciliopsis occur together in desert streams of Sonora, Mexico. Their coexistence has been explained in terms of niche partitioning for food and space. We examined predatory behavior that might influence niche relationships, and found significant differences among two coexisting sperm-dependent clonal strains and their two sexual progenitors. Handling time and prey manipulation of freeswimming (Artemia) and benthic (chironomid larvae) prey differed significantly among sexual and clonal strains. Analyses of gut contents from field-collected fish revealed that the laboratory estimates of predatory efficiency were related to their feeding behavior in nature. Behavior differences, such as those described herein, contribute to our understanding of the mechanisms of unisexual/bisexual coexistence in Poeciliopsis.
\end{abstract}

\section{Introduction}

All-female reproduction of an asexual population provides a twofold demographic advantage when compared with a sexual population that must pay the cost of producing males (Williams 1975; Maynard Smith 1978). Given this strong advantage, attempts to explain the persistence of sexuality in mixed reproductive complexes have often focused on differences in niche characteristics of sexual and asexual populations (Ghiselin 1974; Bell 1982). According to these models, individual clones might outcompete sexuals for resources on which each clone is specialized, but a broadly adapted sexual population, composed of many genotypes, can persist by ex-

\footnotetext{
* Current address: Savannah River Ecology Laboratory, Drawer E, Aiken, SC 29801, USA

** Current address: Biology Department, University of MichiganFlint, Flint, MI 48502, USA

*** Current address: 3255 Lee Rd., Shaken Heights, OH 44120 , USA

Offprint requests to: R.C. Vrijenhoek
}

ploiting the remaining uncontested resources. As long as the assemblage of clones does not completely eclipse the resource spectrum used by the sexuals, both reproductive modes can persist (Bell 1982; Case and Taper 1986). Within this context, the "frozen niche-variation" model (Vrijenhoek 1979, 1984) was postulated to explain coexistence among members of an assemblage of genetically distinct clones and their sexual relatives. It is based on two premises: (1) different clones arise independently from genetically variable sexual ancestors; and (2) each new clone freezes and faithfully replicates a unique genotype that affects microhabitat use and resource consumption. Given sufficient opportunity for new clonal origins, interclonal selection should produce a structured assemblage of clones with reduced ecological overlap, each clone exploiting a different portion of the ancestral sexual niche. Differential resource use should facilitate coexistence among clones, as well as coexistence with a broad-niched sexual ancestor.

Studies with clonal and sexual fishes of the genus Poeciliopsis (Atheriniformes:Poeciliidae) have proven useful in testing the assumptions and predictions of the frozen niche-variation model. The key premises of the model (e.g., multiple origins and frozen phenotypic differences among clones) have been tested and verified in controlled laboratory studies (Wetherington et al. 1987, 1989). Despite their potential twofold reproductive advantage, sexual and asexual strains of Poeciliopsis coexist as mixed reproductive complexes in the desert streams of northwestern Mexico. Field and laboratory studies have suggested that niche partitioning is partially responsible for this coexistence (Vrijenhoek 1978; Schenck and Vrijenhoek 1986, 1989). Hence, the inferences of the frozen niche-variation model have some empirical support in these fish.

Although inheritance is strictly clonal, all-female forms of Poeciliopsis require sperm from an appropriate sexual host to reproduce. Thus, an alternative model for coexistence is based on their sperm dependence (Moore and McKay 1971; Moore 1976). However, recent theoretical examinations of this alternative model 
found that frequency-dependent mate discrimination favoring sexuals is only necessary for coexistence in situations of complete niche overlap (Kirkendall and Stenseth 1990). An additional problem of the sperm-dependence model is that it fails to explain the coexistence of multiple clones in a mixed complex (Vrijenhoek 1978), unless males discriminate only against the most common clones (Keegan-Rogers and Schultz 1984). Kirkendall and Stenseth (1990) concluded that reduced niche overlap, as invoked by the frozen niche-variation model, may be the primary factor stabilizing sperm-dependent clonal/sexual complexes.

Evidence for ecologically structured assemblages of Poeciliopsis emerged from field studies of the monacha complex which includes the sexual species $P$. monacha plus two allotriploid clones of the biotype P. 2 monachalucida (Vrijenhoek 1978; Schenck and Vrijenhoek 1986, 1989). However, less is known about the lucida complex which inhabits the downstream sunny portion of this stream. This complex includes the sexual host $P$. lucida plus two allodiploid hemiclones of the hybridogenetic biotype $P$. monacha-lucida, $M L / V I I$ and $M L / V I I I$, which arose endemically as hybrids between $P$. monach $a$ and P. lucida (Vrijenhoek et al. 1978; Quattro et al. 1991). Hemiclonal reproduction occurs through a process called hybridogenesis (Schultz 1969). Only the haploid monacha $(M)$ genome of the $M L$ hybrids is transmitted without recombination to their eggs; the paternal lucida $(L)$ genome is discarded during oogenesis (Cimino 1972). Each generation, a new $L$ genome is acquired by true fertilization of the haploid $M$ ova by sperm from $P$. lucida males.

A field study of the microspatial distributions of members of the lucida complex also revealed evidence for spatial segregation among hemiclonal and sexual forms, thus decreasing direct competition (Schenck and Vrijenhoek 1986). However, the study did not examine differences in diets. In what follows, we describe a coordinated field and laboratory study of feeding behavior of $M L / V I I$ and $M L / V I I I$ and their sexual progenitors from the Arroyo de Jaguari, $P$. monacha and P. lucida. The results are used to test the main prediction of the frozen niche-variation model - that coexisting clones have frozen distinct genotypes that show reduced ecological overlap with one another and with the sexual progenitors.

\section{Materials and methods}

Fish strains. We examined fish collected from a headwater tributary of the Rio Fuerte (Arroyo de Jaguari, ca. Agua Caliente, Sonora, Mexico). The two unisexual strains (accession codes: S68-4 $M L$ $V I I$ and $\mathrm{T} 70-3 \mathrm{ML} / V I I I)$ were perpetuated as laboratory strains for 10-12 years prior to the present experiments. In the current study, the paternal $L$ genome of $M L$ hybrids was standardized by mating each strain to an inbred, isogenic strain of $P$. lucida (S68-4 PC; Angus and Schultz 1983; Vrijenhoek et al. 1978). This procedure rendered the two $M L$ hemiclones identical for their paternal $L$ genomes, while preserving differences due to their $M$ genomes. The sexual strains of $P$. monacha and $P$. lucida were secondgeneration stocks reared from outcrossed wild populations.
Behavioral experiments. The behavioral experiments consisted of two independent predation efficiency tests (Werner 1977), one using live chironomid larvae to stimulate naturally occurring benthic prey, and the other using live Artemia aduits to simulate freeswimming invertebrate prey. Chironomid larvae constitute a significant fraction of the natural diet of Poeciliopsis (Schenck and Vrijenhoek 1989). Although Artemia are not natural prey for these fish, many free-swimming invertebrates, such as copepods and ostracods, are eaten. Estimation of differences in feeding efficiency on invertebrate prey are important for two reasons. First, the complexities of capturing and consuming live prey add multiple niche dimensions upon which these fish strains may partition resources. Second, although invertebrates do not comprise the majority of the food consumed in all four strains, these energy-rich resources may comprise the majority of available calories ingested by these fish (Schenck and Vrijenhoek 1989).

All experimental fish were born and reared in the laboratory and maintained in 40-1 aquaria. The fish were fed daily rations of Purina trout chow plus live chironomids and brine shrimp to condition them to live food. Fish within the same size range (23$29 \mathrm{~mm}$ ) were used for both experiments. Temperature was maintained at $24^{\circ} \pm 1^{\circ} \mathrm{C}$ throughout the experimental period. All behavioral observations were taken from behind a cardboard blind, and events were timed with a four-channel digital stopwatch.

In the chironomid experiment, nine fish of each of the four strains were examined for prey handling-time. Fish were starved for 1 day prior to each feeding trial and conditioned by exposing them ten times to the testing procedure (described below). Directly following conditioning, four experimental trials were performed with each fish. Trials were conducted in 40-1 aquaria partitioned into two halves with an opaque plastic divider. An individual fish was placed in one half of the observation tank and two chironomid larvae were placed in diagonally opposing corners of the other half (Fig. 1). All fish were allowed to acclimate to the experimental tank for 3 min before the divider was lifted and measurements were taken. Handling time was defined as the time (in seconds) between striking a chironomid and initiating search for the second chironomid. To avoid entrainment of searching behavior among the four trials per individual, placement of chironomid larvae was randomly assigned between alternate patterns within the test chamber (configurations $\mathrm{A}$ and $\mathrm{B}$ in Fig. 1). Care was taken to standardize chironomid size in all trials.

In contrast to the chironomid larvae, Artemia were more rapidly consumed. It was not possible to separate time spent handling prey from search and capture. Thus, we calculated the average time spent per prey item as the time between the first strike on the first brine shrimp and the first strike on the sixth shrimp, divided by five. Four trials were performed with between 8 and 11 fish for each of the four strains. The trials were conducted in a 1-1 aquarium with opaque walls. Observations were made from above using a $45^{\circ}$ angled mirror. Conditioning procedures were as described for the chironomid experiment. Each trial consisted of the presentation of six adult Artemia introduced through a largebore pipette. Again, care was taken to present similarly sized prey to all fish in all trials.

Dietary analysis. To establish the consistency of the laboratory behavioral experiments with natural feeding habits, we examined the gut contents of wild-caught $P$. monacha, $P$. lucida, $M L / V I I$, and $M L / V I I I$. Fish were collected from the Río Fuerte (Arroyo de Jaguari, ca. Agua Caliente) in December 1978 and June 1980. Immediately upon capture, specimens were frozen on dry ice to prevent evacuation of the gut. Specimens were subsequently stored at $-60^{\circ} \mathrm{C}$. To electrophoretically identify specimens in the laboratory, we removed the eyes from each fish, measured its standard length (SL), and labelled the carcass for refreezing. Earlier electrophoretic studies revealed that these strains differ at many loci (see Vrijenhoek et al. 1978 for methods). For the purposes of this study, these strains could be unequivocally separated using the products of only two gene loci, Ldh-1 (E.C. \#1.1.1.27) and Pgd (E.C. \#1.1.1.44). 
Configuration A

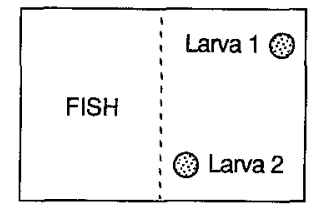

Configuration B

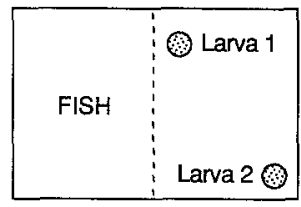

Fig. 1. Experimental design for the chironomid experiment. Dashed line represents the opaque plastic divider

Gut contents were examined from a subset of wild fish that were similar in size to those of the behavioral experiments ( $S L=23$ $39 \mathrm{~mm}$ ). Examinations were performed blindly with respect to the identity of the fish strain. The alimentary canal of Poeciliopsis is tubular, without a discrete stomach. The entire gut was removed from freshly thawed fish and microscopically examined using transillumination. Its transparency facilitated quantification of food categories. The gut was treated as a simple cylinder and the approximate volume of each food type was measured as the proportion of the gut length occupied by discrete packets of invertebrates, algae, sand, and detritus. The gut was subsequently dissected and inidividual food items were identified to the taxonomic level of family.

Statistical methods. All data were analyzed using the General Linear Models, Frequency, 1-Way Nonparametric, and Univariate procedures of the SAS statistical package (SAS 1982). Predation efficiency estimates were averaged to give a single measurement per individual. An analysis of covariance was performed on the log-transformed feeding efficiencies to correct for the effects of differences in size among the various fish strains. Slope heterogeneity was not significant and log-transformed data did not significantly deviate from normality. Pairwise comparisons were made between the least-squares means using a Bonferroni correction for multiple comparisons (Snedecor and Cochran 1980).

One-way nonparametric analyses of variance were made for differences in consumption of the four broad food categories. Each fish was scored according to the percent (by volume) of each food item found in its gut contents. These scores were then compared with a Kruskal-Wallis test for heterogeneity among strains (Hollander and Wolfe 1973).

\section{Results}

\section{Behavioral experiments}

A bivariate plot (Fig. 2) illustrates the relationship between predation efficiency of fish fed Artemia vs. those fed chironomid larvae. Mean predation efficiencies on the two food items were highly correlated $(r=0.996$; $P<0.01$ ), indicating that both behavioral measures were basically quantifications of overall predation efficiency on invertebrate prey. Predation efficiency on chironomid larvae differed significantly among the four strains (Table 1 A). Pairwise comparisons showed that $P$. lucida and $M L / V I I$ had longer handling-times than $P$. monacha and $M L / V I I I$. Predation efficiency on Artemia also differed significantly among strains (Table 1B). P. lucida was the least efficient predator, and $M L / V I I I$ was the most efficient.

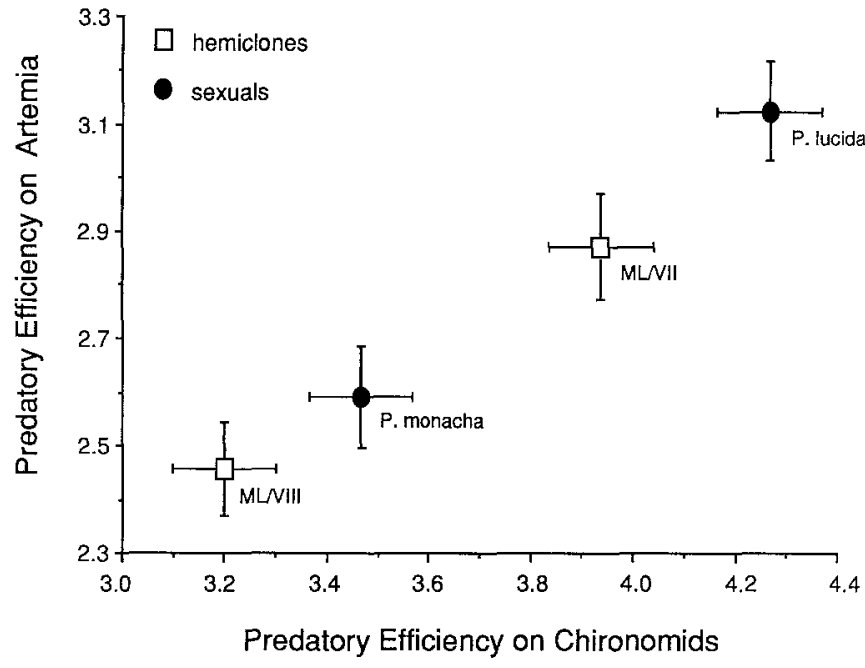

Fig. 2. Bivariate plot of prey handling times of four Poeciliopsis strains fed Artemia adults and chironomid larvae. Handling times are expressed in terms of least squares means of natural-log transformed handling times for both prey items (see text). Solid circles represent the the two sexual strains, open squares represent the two allodiploid hemiclones. Error bars portray $1 \mathrm{SE}$

Table 1. ANCOVA of feeding experiments on Poeciliopsis

A) Chironomid Experiment (average handling time)

\begin{tabular}{|c|c|c|c|c|c|}
\hline Source & $d f$ & Mean square & $F$ & $P$ & \\
\hline Length & 1 & 8.5830 & 94.01 & 0.0001 & \\
\hline Strain & 3 & 2.0026 & 21.93 & 0.0001 & \\
\hline Error & 31 & 0.0913 & & & \\
\hline Strain & & P. lucida & $M L / V I I$ & P. monacha & $M L / V I I I$ \\
\hline LSM(ln) & & 4.266 & 3.938 & 3.466 & 3.199 \\
\hline
\end{tabular}

B) Artemia Experiment (average time per item)

\begin{tabular}{lrllll}
\hline Source & $d f$ & Mean square & $F$ & $P$ \\
\hline Length & 1 & 3.8292 & 49.50 & 0.0001 & \\
Strain & 3 & 0.8536 & 11.03 & 0.0001 & \\
Error & 32 & 0.0774 & & & \\
\hline Strain & & P. lucida & ML/VII & P. monacha & ML/VIII \\
LSM(ln) & & 3.126 & 2.871 & 2.592 & 2.456 \\
& & & & & \\
\hline
\end{tabular}

Underlinded LSM's (least square means of natural-log transformed data) are not significantly different $(P>0.05$ after Bonferroni correction)

Increased handling time for $P$. lucida was primarily due to its need to reorient the prey before swallowing. On average, $P$. lucida needed to strike and release the Artemia more than three times prior to swallowing $($ mean $=3.59 ; \mathrm{SE}=0.22)$. The other strains struck and released prey fewer than once on average $(M L / V I I$ : 
mean $=0.40, \mathrm{SE}=0.14 ; M L / V I I I:$ mean $=0.17, \mathrm{SE}=$ $0.06 ;$ P. monacha $:$ mean $=0.29, \mathrm{SE}=0.14$ ).

\section{Natural diets}

The relative predation efficiencies of the four strains were concordant with the relative proportions of invertebrate prey consumed in their natural environment (Fig. 3). During the end of dry season (June 1980), when fishes are forced into small residual pools, those strains showing higher predatory efficiency in the laboratory experiment showed an identical rank ordering of proportion of invertebrates consumed in their natural diets. Significant differences among strains in the proportion of total invertebrates eaten were largely attributable to the extreme insectivory of hemiclone $M L / V I I I$, particularly with regard to chironomid larvae (Kruskal-Wallis $\left.\chi^{2}(3)=8.57 ; P<0.05\right)$. Chironomid larvae also constituted the majority of the invertebrate prey in $P$. lucida and $M L / V I I$. However, chironomids constituted a smaller proportion of $P$. monacha's diet which contained a greater diversity of other invertebrates (e.g., ostracods and larval dytiscids, odonatés, and stratiomyiids). Consumption of algae differed among strains $\left(\chi^{2}(3)=13.77\right.$, $P<0.005)$ with $P$. monacha obtaining the most algae and $P$. lucida the least. Finally, consumption of sand (indicative of deposit feeding) was heterogeneous among strains $\left(\chi^{2}(3)=19.47, P<0.001\right)$ with $P$. lucida and $M L$ VII having the highest proportion of sand in their diets.

Following the rainy season (mid-July to October), water levels are higher, streams become scoured of filamentous algae, and insect diversity changes reflecting an increase in abundance of insects that favor flowing water (e.g., Ephemeroptera and simuliids). The congruence between predation efficiency and diet observed in June 1980 was less evident in the post rainy-season sample of December 1978 (Fig. 3B). Consumption of invertebrates was still heterogeneous among strains $\left(\chi^{2}\right.$ $(3)=19.48, P<0.001$ ), and $P$. lucida was still the least efficient predator; however, $P$. monacha had the most abundant and diverse invertebrate diet, consuming nearly equal quantities of chironomids, Ephemeroptera, and other invertebrates (e.g., hydrocarinid mites and larval simuliids, ceratopogonids, and elmids). Although hemiclones $M L / V I I$ and $M L / V I I I$ captured intermediate quantities of invertebrates, $M L / V I I$ consumed mainly chironomids and $M L / V I I I$ consumed mainly larval Ephemeroptera, which are considerably larger. The proportion of filamentous algae in the diet exhibited the same rank ordering as in the June 1980 sample, with $P$. monacha again being the most herbivorous, but differences were not statistically significant. Finally, the proportion of sand in the diet was highly heterogeneous among strains $\left(\chi^{2}(3)=19.47, P<0.001\right)$, with $P$. lucida, $M L / V I I$, and $M L / V I I I$ consuming more than $50 \%$ sand, whereas $P$. monacha consumed only $23 \%$ sand.

Overall, those species with intermediate feeding efficiency in the laboratory experiment $(P$. monacha and $M L / V I I)$ seemed to forage more opportunistically, ingesting a smaller proportion of invertebrates in the dry

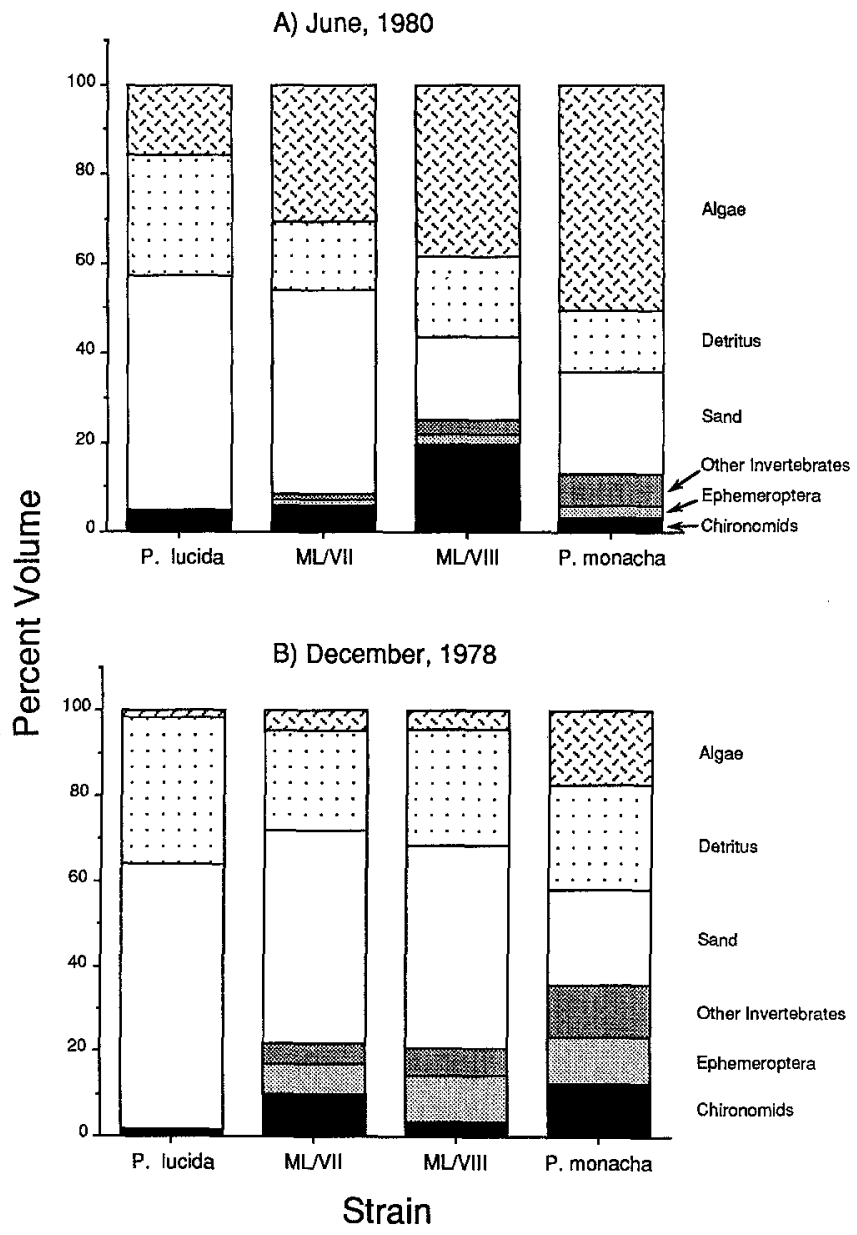

Fig. 3A, B. Mean gut volumes. The invertebrate category was subdivided into chironomid larvae, ephemeroptera larvae, and other invertebrates (ostracods, nematodes, hydracarina, Trichoptera, $\mathrm{Co}-$ leoptera, and Simulium larvae). A Gut contents of 15 fish of each strain collected during the dry season (June 1980); B gut contents of 18 fish of each strain collected during the rainy season (December 1978

Table 2. Percent overlap of resource use of the four strains of Poeciliopsis in the wet and dry seasons. Values above the diagonal are for the dry season (June 1980) and those below the diagonal are for the wet season (December 1978)

\begin{tabular}{lllll}
\hline Strain & P. lucida & ML/VII & ML/VIII & P. monacha \\
\hline P. lucida & 1.000 & 0.813 & 0.570 & 0.560 \\
ML/VII & 0.767 & 1.000 & 0.728 & 0.790 \\
ML/VIII & 0.780 & 0.909 & 1.000 & 0.727 \\
$P$. monacha & 0.503 & 0.725 & 0.721 & 1.000 \\
\hline
\end{tabular}

season and a larger proportion after the wet season. On the other hand, species with extreme predatory efficiencies in the laboratory were relatively consistent across seasons. P. lucida ate the fewest invertebrates and the most sand and detritus during both seasons. Hemiclone $M L / V I I I$ consumed a relatively consistent proportion of invertebrates across both seasons.

Schoener's (1970) index of resource overlap can be used to describe the degree of overlap in diet between 
pairs of coexisting species (Table 2). Average dietary overlap was roughly the same in the December 1978 $(73 \%)$ and June $1980(69 \%)$ samples. The lowest overlap across both seasons occurred for the two sexual species, $P$. monacha and $P$. lucida. The greatest overlap occurred between the two hemiclones during December; however, that overlap dropped $18 \%$ during the dry season when insects were less abundant. On average, the most predacious strain, $M L / V I I I$, exhibited greater overlap with other strains during December $(80 \%)$ than during June $(68 \%)$. This reduction in resource overlap was due to $M L / V I I T$ 's continued consumption of insects in the dry season even though insects were rare.

\section{Discussion}

According to the frozen niche-variation model, coexisting clonal lineages have distinct genotypes that produce phenotypic distributions with reduced ecological overlap with one another and their sexual progenitors (Vrijenhoek 1979,1984$)$. This study identified significant differences in predatory behavior between coexisting hemiclones of P. monacha-lucida and females of their sexual host, $P$. lucida, and demonstrated that these differences were consistent with natural food habits. P. lucida was the least efficient predator in the laboratory and showed the lowest consumption of invertebrates in the field. The hemiclone with intermediate feeding efficiency in the laboratory, $M L / V I I$, ingested a high proportion of invertebrates after the rainy season when aquatic insects were more abundant, but became more detritivorous during the dry season. The most efficient predator in the laboratory study, $M L / V I I I$, obtained a consistently large proportion of invertebrates during both seasons. The increased volume of water following the rainy season may minimize spatial interactions among these strains, while competitive interactions may increase during the dry season when fish are forced into small residual pools and when aquatic insects are less abundant (Thibault 1974a). Thus, differences among strains in resource use during the dry season may primarily determine their distributions. This finding is especially pertinent for $M L / V I I$ and P. lucida which exhibit the greatest dietary overlap during the dry season, both being primarily deposit feeders. A reanalysis of earlier data (Schenck and Vrijenhoek 1986, Table 4) reveals that natural frequencies of these strains are negatively correlated on a scale related to local habitat patchiness $(r=-0.467 ; P<0.05)$. No significant correlations, negative or positive, exist with the other combinations, $P$. lucida vs. $M L / V I I I$ or $M L / V I I$ vs $M L / V I I I$, in this complex. Apparently, spatial segregation serves as a means for decreasing direct competition between $P$. lucida and $M L / V I I$ during the dry season.

Hemiclone $M L / V I I I$, on the other hand, is less affected by local habitat patchiness (Schenck and Vrijenhoek 1986). This hemiclone tends to be very aggressive in the laboratory compared to $M L / V I I$ and $P$. lucida (Keegan-Rogers and Schultz 1984). For the behavioral traits examined to date, hemiclone $M L / V I I I$ appears to be an aggressive carnivore, which contrasts with the relatively docile, deposit feeding habits of $P$. lucida and $M L /$ $V I I$. Hemiclone $M L / V I I T$ 's predation efficiency allows for the consumption of a high proportion of invertebrate prey, even during the dry season when most aquatic insects are rare. Perhaps the combination of $M L / V I I T$ 's aggressive behavior and its efficiency with invertebrate prey facilitates coexistence with the deposit feeders, which might explain the absence of negative spatial correlations with the other members of the lucida complex (Schenck and Vrijenhoek 1986).

Poeciliopsis monacha was included in this study so that the feeding characteristics of both sexual ancestors of the P. monacha-lucida strains could be compared. Clearly $P$. monach $a$ and $P$. lucida represent distinct feeding strategies, as they exhibited the least dietary overlap during both seasonal samples. However, ecological interactions between the monacha and lucida complexes are probably minimal. The monacha complex is not abundant in downstream portions of the Arroyo de Jaguari where the lucida complex predominates (Thibault 1974a; Schenck and Vrijenhoek 1986). The monacha complex favors rocky pools and springs in headwater portions of this stream and its tributaries. P. monacha's distinct resource use reflects adaptation to a different environment and coexistence with a different set of hybrid clones.

Nevertheless, previous field studies of diets and spatial distributions of the monacha complex reveal a set of relations similar to those reported herein for the lucida complex. Both triploid clones tend to feed on drifting invertebrates from flowing water, whereas $P$. monacha females tend to feed in still-water pools (Schenck and Vrijenhoek 1986, 1989). Clone $M M L / I$ substantially increases the proportion of invertebrates in its diet when feeding in currents, and its shows no large-scale pattern of spatial segregation with respect to other member of this complex. Reanalysis of previously published data (Vrijenhoek 1978, Fig. 1) reveals that $M M L / I$ s frequencies are not significantly correlated with either $P$. monacha or $M M L / I I$. However, P. monacha and $M M L / I I$ segregate on a spatial scale related to stream order and primary productivity. Their frequencies are strongly negatively correlated $(r=-0.912 ; P<0.01)$, with $M M L / I I$ most frequent in sunny, productive habitats, and $P$. monach $a$ females dominating austere bedrock pools in shady upstream areas. As observed in the present study of the lucida complex, strains that overlap strongly in diet tend to segregate in the spatial dimension.

According to the frozen niche-variation model, differences among clones are frozen from variability that exists in the sexual ancestors. If clonal phenotypes reflect only the additive properties of fixed genomic differences between the sexual ancestors, then allodiploid and allotriploid strains should be arrayed between $P$. monacha and $P$. lucida according to genomic dosages (Thibault 1974a, b; Moore 1984). Although diploid and triploid strains of Poeciliopsis exhibit dentitional phenotypes, egg sizes, and cannibalistic behavior with the predicted genomic dosage patterns (Schultz 1969; Thibault 1974b), the predatory characteristics observed in this study did not 
fit these patterns. Hemiclone $M L / V I I I$ was a more efficient predator than either sexual ancestor. Because this behavior was observed in laboratory strains with standardized (isogenic) paternal genomes, the field data on natural diets were especially relevant. Even when the paternal lucida genome was substituted freely in natural hybridogens, this hemiclone still exhibited a highly predacious diet. Therefore, its predatory behavior probably does not merely reflect additive variance, but instead may be determined by nonadditive effects. $M L / V I I I$ might have captured extreme monacha genes that behave dominantly with respect to the lucida genome in this hybrid. Alternatively, the phenotypically extreme strains might have evolved subsequent to their origins as hybrid clonal forms. Although post-formational mutations are potentially deleterious (Leslie and Vrijenhoek 1978, 1980), some might contribute to the adaptive potential of clonal lineages (Vrijenhoek and Schultz 1974; Lynch 1984).

In summary, coexisting sexual and clonal forms of Poeciliopsis tend to differ from one another in their use of food or spatial resources. Although the predatory behaviors observed in this study do not solely determine niche relationships among these fish, they clearly correspond with diets in their natural habitats. As observed for many closely related species (Schoener 1974), taxa that overlap substantially on the food resource dimension (e.g., $M L / V I I$ vs. P. lucida) often segregate on the habitat dimension. Combined laboratory and field studies suggest that such niche relationships also facilitate clonal/sexual coexistence as predicted by the food and spatial resource heterogeneity models (Ghiselin 1974; Vrijenhoek 1979, 1984; Bell 1982; Case and Taper 1986).

Acknowledgements. We thank P. Smouse, R. Lima, S. Schutz, J. Quattro, P. Leberg, and J.D. Baldwin for critically reviewing the manuscript. We are indebted to R.J. Schultz for providing the unisexual strains used in the laboratory study. Research was supported by grants from the National Science Foundation (DEB8212150 and BSR 86-00661).

\section{References}

Angus RA, Schultz RJ (1983) Meristic variation in homozygous and heterozygous fish. Copeia 1983:287-299

Bell G (1982) The masterpiece of nature. The evolution and genetics of sexuality. University of California Press, Berkeley, CA

Case TJ, Taper MC (1986) On the coexistence and coevolution of asexual and sexual competitors. Evolution 40:366-387

Cimino MC (1972) Egg production, polyploidization and evolution in a diploid all-female fish of the genus Poeciliopsis. Evolution $26: 294-306$

Ghiselin MT (1974) The economy of nature and the evolution of sex. University of California, Berkeley, CA

Hollander M, Wolfe DA (1973) Nonparametric statistical methods. Wiley, New York

Keegan-Rogers V, Schultz RJ (1984) Differences in courtship aggression among six clones of unisexual fish. Anim Behav 32:1040-1044

Kirkendall LR, Stenseth NC (1990) Ecological and evolutionary stability of sperm-dependent parthenogenesis: Effects of partial niche overlap between sexual and asexual females. Evolution $44: 698-714$
Leslie JF, Vrijenhoek RC (1978) Genetic dissection of clonally inherited genomes of Poeciliopsis I. Linkage analysis and preliminary assessment of deleterious gene loads. Genetics 90:801-811

Leslie JF, Vrijenhoek RC (1980) Consideration of Muller's ratchet mechanism through studies of genetic linkage and genomic compatibilities in clonally reproducing Poeciliopsis. Evolution 34:1105-1115

Lynch M (1984) Destabilizing hybridization, general-purpose genotypes and geographical parthenogenesis. Q Rev Biol 59:257290

Quattro JM, Avise JC, Vrijenhoek RC (1991) Molecular evidence for multiple origins of hybridogenetic fish clones (Poecilidae: Poeciliopsis). Genetics 127:391-398

Maynard Smith J (1978) The evolution of sex. Cambridge University Press, Cambridge

Moore WS (1976) Components of fitness in the unisexual fish Poeciliopsis monacha-occidentalis. Evolution 30:564-578

Moore WS (1984) Evolutionary ecology of unisexual fishes. In: Turner BJ (ed) Evolutionary genetics of fishes. Plenum Press, New York, pp 329-398

Moore WS, McKay FE (1971) Coexistence in unisexual-bisexual species complexes of Poeciliopsis (Pisces:Poeciliidae). Ecology 52:791-799

SAS (1982) SAS user's guide: Statistics. SAS Institute, Cary, NC

Schenck RA, Vrijenhoek RC (1986) Spatial and temporal factors affecting coexistence among sexual and clonal forms of $P_{\text {oeci- }}$ liopsis. Evolution 40:1060-1070

Schenck RA, Vrijenhoek RC (1989) Coexistence among sexual and asexual forms of Poeciliopsis: Foraging behavior and microhabitat selection. In: Dawley R, Bogart J (eds) Evolution and ecology of unisexual vertebrates. State University of New York Press, Albany New York, pp 39-48

Schoener TW (1970) Non-synchronous spatial overlap of lizards in patchy habitats. Ecology $51: 408-418$

Schoener TW (1974) Resource partitioning in ecological communities. Science 157:1564-1567

Schultz RJ (1969) Hybridization, unisexuality and polyploidy in the teleost Poeciliopsis (Poeciliidae) and other vertebrates. Am Nat 103:605-619

Snedecor GW, Cochran WG (1980) Statistical methods. Iowa State University Press, Ames, IA

Thibault RE (1974a) The ecology of unisexual and bisexual fishes of the genus Poeciliopsis: A study in niche relationships. Ph.D. Thesis, University of Connecticut, Storrs

Thibault RE (1974b) Genetics of cannibalism in viviparous fish and its relationship to population density. Nature 251:138-140

Vrijenhoek RC (1978) Coexistence of clones in a heterogeneous environment. Science 199:549-552

Vrijenhoek RC (1979) Factors affecting clonal diversity and coexistence. Am Zool 19:787-797

Vrijenhoek RC (1984) Ecological differentiation among clones: the frozen niche variation model. In: Wöhrmann $\mathrm{K}$, Loeschke V (eds) Population biology and evolution. Springer, Berlin Heidelberg New York, pp 217-231

Vrijenhoek RC, Schultz RJ (1974) Evolution of trihybrid unisexual fish (Poeciliopsis, Poeciliidae). Evolution 28:205-319

Vrijenhoek RC, Angus RA, Schultz RJ (1978) Variation and clonal structure in a unisexual fish. Am Nat 112:41-55

Werner EE (1977) Species packing and niche complementarity in three sunfishes. Am Nat 111:553-578

Wetherington JD, Kotora KE, Vrijenhoek RC (1987) A test of the spontaneous heterosis hypothesis for unisexual vertebrates. Evolution $41: 721-731$

Wetherington JD, Weeks SC, Kotora KE, Vrijenhoek RC (1989) Genotypic and environmental components of variation in growth and reproduction of fish hemiclones (Poeciliopsis:Poeciliidae). Evolution $43: 635-645$

Williams GC (1975) Sex and evolution. Princeton University Press, Princeton, NJ 